\title{
Gestión del Conocimiento a través del modelo de Socialización, Exteriorización, Combinación e Interiorización (SECI): En las bibliotecas de la Universidad Nacional Abierta Centro Local Falcón
}

doi: 10.33264/rpa.202001-04

MSc. Fernando Antonio Salas Granado

Escuela de Bibliotecología y Archivología. Universidad Central de Venezuela

Facultad de Humanidades y Educación. Universidad Central de Venezuela

\section{Resumen}

Si el conocimiento de un individuo se inhibe, en relación con otros miembros de la organización, el conocimiento de esta será mucho menos efectivo. Para Nonaka y Takeuchi (1995), una de las tareas importantes de la gestión del conocimiento es la de facilitar las interacciones entre los miembros de la organización, se trata de un proceso circular y en espiral, que consta de una serie de subprocesos: creación de conocimiento, adopción de conocimiento, distribución de conocimiento y revisión de conocimiento.

La concepción de la aplicación de la gestión del conocimiento a través del Modelo de Socialización, Exteriorización, Combinación e Interiorización (SECI) de Nonaka y Takeuchi (1995), dirigido al personal de las Bibliotecas del Centro Local Falcón, es de gran importancia en el proceso de enseñanza-aprendizaje, aunado al desarrollo de modelos formativos más flexibles e innovadores para la captación y transmisión del conocimiento. El talento humano, es la base del conocimiento y la experiencia laboral es de gran valor agregado, con la finalidad de incentivar el aprendizaje. La modalidad de investigación descriptiva, apoyada en un estudio de campo, la población quedó conformada por tres Asistentes de Biblioteca, una Secretaria y un Coordinador de Biblioteca, pertenecientes al Centro Local Falcón de la Universidad Nacional Abierta. Para recabar la información se utilizó como técnica la encuesta y como instrumento un cuestionario conformado por 25 preguntas abiertas. En el desarrollo de este artículo esbozaremos este Modelo con las siglas utilizadas por Nonaka y Takeuchi (1995): 'SECl'.

Palabras claves: Gestión del conocimiento, Socialización, Exteriorización, Combinación, Interiorización, SECI, UNA, Centro Local Falcón. 


\begin{abstract}
If the knowledge of an individual is inhibited, in relation to other members of the organization, the knowledge of this will be much less effective. For Nonaka and Takeuchi (1995), one of the important tasks of knowledge management is to facilitate interactions between the members of the organization, it is a circular and spiral process, consisting of a series of sub-processes: creation of knowledge, adoption of knowledge, distribution of knowledge and review of knowledge.
\end{abstract}

The conception of the application of knowledge management through the Model of Socialization, Externalization, Combination and Internalization (SECI) by Nonaka and Takeuchi (1995), aimed at the staff of the Falcón Local Center Libraries, is of great importance in the teaching-learning process, coupled with the development of more flexible and innovative training models for the capture and transmission of knowledge. Human talent is the basis of knowledge and work experience is of great added value, in order to encourage learning. The descriptive research modality, supported by a field study, the population was made up of three Library Assistants, a Secretary and a Library Coordinator, belonging to the Falcón Local Center of the National Open University. To collect the information, the survey was used as a technique and a questionnaire made up of 25 open questions as an instrument. In the development of this article we will outline this Model with the acronyms used by Nonaka and Takeuchi (1995): 'SECI'.

Keywords: Knowledge management, Socialization, Externalization, Combination, Internalization, SECI, UNA, Centro Local Falcón.

\title{
Introducción
}

La gestión del conocimiento en las bibliotecas se tiene que caracterizar por su capacidad para generar, absorber, analizar, distribuir y gestionar el volumen de información existente en su interior, transformándola en conocimiento. Una vez delimitado el servicio de gestión del conocimiento es necesario generar nuevos servicios mediante soportes informáticos, por lo que se estima que para poder seguirla es preciso generar continuamente nuevas ideas e innovación, la cual para el caso de las Bibliotecas del Centro Local Falcón de la Universidad Nacional Abierta, puedan guiarse a través de las distintas experiencias del personal que labora en ellas. Las bibliotecas universitarias han cambiado con el transcurrir de los años, lo que anteriormente se consideraba un depósito de libros se ha transformado en un espacio vivo y dinámico, cuya misión primordial es servir de centro informativo, documental y de gestión cultural a la comunidad. 
Cada individuo tiene su semblanza y cultura personal, no necesariamente debe saber leer y escribir para ser culto o por lo menos hacer el intento de ocultar el desconocimiento de otras áreas. Tácitamente, cada cerebro pensante, posee técnicas inmemorables de adaptación en su medio, experiencias defensivas heredadas de un centenar de convivencias con las circunstancias que lo rodean; los valores morales, espirituales, arraigados con la sustancia misma de su ser y que se expresan en música, en sus corridos, en sus refraneros, en su calendario, en su comida, que se transmite genéticamente de generación en generación, siempre habrá un legado cultural.

Anteriormente las bibliotecas se preocupaban por conservar el patrimonio escrito, pasando por la misión más tradicional de biblioteca-estudio, soporte de la vida académica e investigativa del individuo; para llegar finalmente a los medios actuales como son la de servir de medio de autoformación permanente, ser mediadora de cultura, constituirse en lugar de encuentros, comunicación, servir de centro de información al ciudadano para poder desenvolverse en la sociedad en que vive.

En la actualidad deben crecer cíclicamente junto con su personal, agilizando las comunicaciones y el conocimiento siendo una fuente de cambios para todos, la cual debe convertirse en una fuerza viva de educación, cultura e información, el libre acceso al conocimiento está determinado en que haya un personal totalmente calificado para orientar a los usuarios, donde exista una fluidez en la comunicación entre las partes que laboran en la biblioteca y que sea perenne la continuidad de trasmisión de conocimientos.

Nonaka y Takeuchi (1995), consideran: "Que el intercambio de experiencias entre los individuos determinan la calidad en cada proceso de producción del conocimiento." El modelo de gestión propuesto por los autores, está conformado por la Socialización, la Exteriorización, la Combinación e Interiorización, denominado Modelo SECI. Por lo tanto, las bibliotecas se caracterizan por representar un servicio cultural y civilizador vital; para satisfacer las necesidades de los individuos y ejercer una amplia influencia en el aprendizaje, la enseñanza y la formación ciudadana. Las bibliotecas son consideradas lugares donde el conocimiento y la cultura fluyen libremente fortaleciendo el desarrollo del sistema educativo. Si el conocimiento es el instrumento para generar nuevo conocimiento y su fuente es la materia gris, entonces con el apoyo de las bibliotecas, donde el usuario no sólo busca una información pertinente, sino las vías para despejar sus dudas, acrecentar su aprendizaje y las herramientas para crear nuevos conocimientos. Matus Sepulveda (2002), argumenta:

La biblioteca es el corazón de una universidad. Ocupa el lugar central y básico, como un recurso que es, porque sirve a todas las funciones de una 
universidad: enseñanza e investigación, la creación de un nuevo conocimiento y la transmisión a la posteridad de la ciencia, la cultura del presente y del pasado.

El Centro Local Falcón de la Universidad Abierta, empieza a funcionar un 27 de noviembre del año 1978, en un local cedido por Fundafalcón en el paseo la Alameda bajo la Coordinación del Profesor Ángel Ferrer; en el año 1979 pasa a ocupar su próxima sede en la calle Churuguara con esquina González y actualmente la sede principal se encuentra en una edificación ubicada en Calle Norte con Avenida Miranda - Coro - Municipio Miranda, hasta la actualidad, por lo tanto, es primordial potenciar la comunicación e intercambio de conocimientos entre el personal de las bibliotecas, lo cual permite crecer exponencialmente la formación, investigación y servicio a la comunidad, donde permanentemente la gestión y difusión de la información sea adaptable a los nuevos cambios que lo requiera la sociedad.

\section{Objetivo General}

Determinar la aplicabilidad de la gestión del conocimiento a través del modelo de Socialización, Externalización, Combinación e Interiorización ( $\mathrm{SECl}$ ), en las bibliotecas de la Universidad Nacional Abierta del Centro Local Falcón.

\section{Metodología}

La modalidad es factible, según lo que establece el Manual de Trabajos de Grado y Tesis Doctórales de la Universidad Pedagógica Experimental Libertador (2016): "Consiste en la investigación, elaboración y desarrollo de una propuesta de un modelo operativo viable para solucionar problemas, requerimientos o necesidades de organizaciones o grupos sociales." De lo anterior se deduce, que la aplicación de la gestión del conocimiento a través del modelo de socialización, externalización, combinación e interiorización al personal bibliotecario es una actividad importante que permite medir el alcance o desempeño de los objetivos propuestos, es decir, determinar si la fluidez del conocimiento tácito que poseen los responsables de las bibliotecas, es transferible, funcional y adecuado a lo que realmente necesitan los usuarios.

\section{Cuerpo del texto}

La Sociedad del Conocimiento se encuentra en un proceso de adecuación a las nuevas condiciones que le impone el entorno nacional e internacional, este proceso de desarrollo apoyado por el Estado, que adquiere, agrega modalidades y elementos adecuados a la realidad actual del país, en la búsqueda de una definición de identidad, capaz de compatibilizar las diferentes vertientes ideológicas de cara al futuro. 
Nos encontramos frente a una oportunidad enmarcada por las nuevas tecnologías de la información y las comunicaciones, su éxito dependerá del planteamiento y su contribución al proceso de valor añadido a los procesos tradicionales en los servicios de información. Sin embargo, se trata de la evolución de bibliotecas convencionales a bibliotecas electrónicas o bibliotecas digitales que deben realizar adelantos y progresos para construir mejoras técnicas que apoyen la gestión del conocimiento y de la innovación organizativa.

Lo que supone crear un juego de sistemas de gestión organizativas efectivas adaptables a las exigencias de la era de biblioteca electrónica para apoyar y reforzar las actividades de la gestión del conocimiento, mejorando los departamentos funcionales y los procedimientos operativos de las bibliotecas, las cuales deben conceder una especial importancia al suministro de servicios para que la gente adquiera conocimiento y pueda ejercer máximas funciones y eficiencia de la información del conocimiento.

Para adoptar una transformación de los diferentes entornos de la biblioteca o centros de información de empresas, gobiernos, organizaciones públicas e instituciones de investigación científica interrelacionadas entre sí y de la información contenida en ella, obliga a la reconceptualización de los métodos, conocimientos y enfoques para la interpretación orgánica y de la funcionalidad de la biblioteca como sistema para la creación de bibliotecas virtuales.

La clasificación del conocimiento que se utiliza y se genera en la gestión del conocimiento conforma cuatro tipos: a) Conocimiento Explícito, b) Conocimiento Implícito, c) Conocimiento Tácito y d) Conocimiento Aplicado.

El conocimiento explícito, se formaliza mediante reglas lógicas (racional) y que de este modo puede hacerse accesible a cualquier persona. El conocimiento explícito puede ser extraído de su contexto original y generalizado de modo que permita a futuros problemas darle una solución. Pueden almacenarse en forma de bases de datos o como conocimientos teóricos, procedimientos, guías de diseño, protocolos de ensayo, entre otros instrumentos institucionales.

El conocimiento implícito se encuentra contextualizado en la forma de trabajar en el día a día, sin ningún tipo de referencia explícita (es informal), cuya existencia se evidencia a través de los resultados. La comunicación entre las personas precisa los niveles de conocimiento implícito equivalentes. 
El conocimiento tácito es el que posee cada individuo acerca de una tarea determinada (habilidad y/o experiencia) esta internalizado al corresponder a cualidades naturales (intuición, creatividad). Este tipo de conocimiento en cada individuo es personal y distinto. La gestión del conocimiento es la encargada de codificar y transformar el conocimiento de cada individuo en la organización, en conocimiento explícito para toda la organización, esta actividad incide en más capacidad y posibilidades para la solución de problemas.

El conocimiento aplicado se refleja en la propia estructura de los resultados tangibles de los proyectos de innovación y en parte la construcción del conocimiento explícito, en base a los conocimientos implícitos y tácitos de las personas que han realizado el proyecto. Este conocimiento se externaliza y clasifica, mediante los informes, documentos, planos, entre otros medios de información y comunicación, y está contenido en los propios productos y procesos.

La coexistencia de estos cuatro tipos de conocimiento en el ámbito del desarrollo de los proyectos de innovación promueve e implica la necesidad de emplear metodologías y herramientas diferentes para lograr una gestión integral del conocimiento. Tanto el conocimiento explicito como el tácito pueden actuar como origen en la creación de nuevo conocimiento.

Los individuos rediseñan su conocimiento para adaptarse a los nuevos cambios existentes, siendo la capacidad de adaptabilidad una característica predominante. La estrategia de aplicación del modelo $\mathrm{SECl}$ desde el punto de vista de la pirámide informacional es factible, en la cual se consideran los cuatro puntos de conversión del conocimiento, datos, información, conocimiento y desarrollo organizacional, estos elementos influyen en el uso de la información, las actitudes y la búsqueda para interiorizar el conocimiento, la capacitación, la experiencia previa, las preferencias personales que permitan desarrollar un contexto dentro de las bibliotecas del Centro Local Falcón y sus Unidades de Apoyo.

Berrocal Berrocal (2001), argumenta que:

"El conocimiento se ha definido como un proceso de aprendizaje para poder hacer algo que antes no se sabía hacer, o para hacerlo mejor; por tanto, no se está ante una actividad puntual, sino ante un proceso continuo que supone añadir valor activo a la información, al filtrarla, sintetizarla, resumirla y utilizarla en el trabajo."

En este orden de ideas propuesto por el autor, el papel del conocimiento en las sociedades han sufrido múltiples cambios y su importancia para los individuos, organizaciones y sociedades se ha intensificado. Así la representación y organización del conocimiento, es considerada como una actividad que está compuesta por la 
identificación, recuperación y la transferencia, aunándose a esto la complejidad del nuevo mundo de la información, relacionado con los altos niveles de producción de conocimiento, por lo que es necesario abarcar los diversos enfoques que se han diferenciado por mucho tiempo en: la ciencia, la filosofía, la práctica, la acción, al hombre y de la naturaleza, ya que, las herramientas para clasificarlas son representaciones que han sido construidas por el hombre, a través de la percepción y la formulación de conceptos abstractos influenciados por la visión del mundo con respecto a la realidad.

El origen de una nueva mentalidad, no fue espontáneamente, ha sido un proceso que ha transcurrido paulatinamente, las primeras manifestaciones ideológicas se concibieron en plena Edad Media, durante la aparición y desarrollo de las instituciones universitarias.

Nuestros antecesores, trataban de formar una sociedad con capacidades y competencias óptimas, para que fuese un ciudadano libre dentro de un estado democrático.

La involución política e inquisidora del feudalismo, acortaban el aprendizaje y la obtención de conocimientos plasmados en pergaminos, libros, papiros, tablillas de barro, que han sido resguardados por los primeros escribanos durante décadas en las abadías o las bibliotecas ocultas dentro de los monasterios. Una vez que se da inicio a las cruzadas, queda demostrado que el mundo occidental, dominado por la iglesia y los señores feudales; no tenían la capacidad de seguir ocultando los datos, información, conocimientos e intercambio de perspectivas culturales y mestizas.

Consecuentemente, los ciudadanos logran salir de la cueva de la ignorancia, se percatan de que el miedo inculcado fervientemente, día a día, era una falacia, no era el sitio más civilizado, ni floreciente del planeta en comparación con otras culturas. Por ejemplo, los cruzados al salir del campo, rumbo a la tierra santa, quedaron deslumbrados por la vista de los edificios, los monumentos y la riqueza cultural que poseía en ese instante Constantinopla.

Un nuevo Cruzado seria Newton, cuando argumentaba: "Si he podido ver a mayor distancia que nadie, ello es porque me he subido sobre los hombros de gigantes", él aludía a los matemáticos Kepler, Galileo, Descartes, etc. En nuestro crecimiento exponencial, le hemos dado valor agregado a la opinión de Newton, a partir de que nunca debemos perder la oportunidad de aprender, enseñar, tener la generosidad de desprendernos de nuestros conocimientos, para que exista una continuidad y otros prosigan con la multiplicación de saberes. 
En este sentido las personas o compañeros de trabajo, prescinden de una posible pasividad, para establecer una relación de actividad y espontaneidad con un ambiente abierto a los estímulos y la capacidad de aprender, participar en el proceso de desarrollo y transmisión de conocimiento, poniéndose de manifiesto la capacidad humana de entender, predecir, procesar y controlar la información como un elemento fundamental para la permanencia, el mejoramiento de la calidad de vida, la división del trabajo y el aporte filosófico que estableció la distinción entre religión, ciencia y la búsqueda del conocimiento.

El conocimiento tácito, aunque es personal y difícil de codificar formalmente, se puede compartir y revelar informalmente mediante la comunicación, a través de un proceso de combinación, verificación y refinación, transformando el conocimiento tácito en anécdotas más explicitas, innovaciones, productos y servicios mejorados, nuevas capacidades de aprendizaje, conformación de un grupo de trabajo con la capacidad de desarrollar nuevo conocimiento al absorber la información disponible de su entorno y transferir su propio aprendizaje a otros.

La ventaja competitiva que poseen las instituciones de educación superior es su capacidad para producir conocimiento entre las personas o equipos de trabajo que contribuyen a un rendimiento superior, con fines específicos. El principal valor del conocimiento, en cualquier organización, es la habilidad para abordar y solucionar asuntos complejos de forma eficiente y creativa. La importancia de la gestión del conocimiento en las bibliotecas, se fundamenta en la conglomeración de datos, complementado con la experiencia, siendo esta una fuente de ventajas competitivas de carácter razonable, son recursos de dominio público y flexibles.

Rodríguez (2012), considera: "La idea es trascender las condiciones actuales a unas mejores". La importancia de la gestión del conocimiento en las bibliotecas, se fundamenta en la conglomeración de datos, complementado con la experiencia, siendo esta una fuente de ventajas competitivas de carácter razonable, son recursos de dominio público y flexibles. Si el conocimiento de un individuo se inhibe, en relación con otros miembros de la organización, será mucho menos efectivo.

Para Nonaka (1995), una de las tareas importantes de la gestión del conocimiento es:

\begin{abstract}
"La de facilitar las interacciones entre los miembros de la organización, se trata de un proceso circular y en espiral, que consta de una serie de subprocesos: creación de conocimiento, adopción de conocimiento, distribución de conocimiento y revisión de conocimiento."
\end{abstract}

Queda establecido, durante este lapso de comunicación que la espiral del conocimiento no es un proceso lineal y secuencial, sino exponencial y dinámico, que 
parte del elemento humano y de su necesidad de contrastar, validar sus ideas y premisas; de esta forma el individuo a través de la experiencia crea conocimiento tácito, el cual conceptualiza, convirtiéndolo en explícito individual, solo cuando se logra compartir a través del diálogo fluido con otro o varias personas que forman parte de un departamento u organización, se convertirá en conocimiento explícito social, una vez alcanzada esta etapa de comunicación se debe interiorizar las experiencias, ideas o acciones de otras personas, lo cual genera una transmisión y percepción cíclica, en donde la comunicación es un factor primordial.

El proceso de creación del conocimiento para Nonaka y Takeuchi (1995), es a través de un modelo de espiral (ver figura 1), en la cual existe una interacción entre el conocimiento tácito y explícito, teniendo una naturaleza dinámica y continúa; este modelo está conformado por:

a.- La Socialización (tácito a tácito), es el proceso de adquirir el conocimiento tácito que es muy personal de un individuo, a través de compartir experiencias directamente con otra, asimilando las destrezas de éste mediante la observación, la imitación y la práctica, estas habilidades se vuelven parte de su base de conocimiento tácito; puesto que este conocimiento es difícil de normalizar, las empresas lo adquieren de sus empleados, de sus clientes y de sus proveedores, trabajando en forma conjunta con ellos.

b.- La Exteriorización (tácito a explícito), es el proceso de convertir conocimiento tácito en explícito, esto se hace compartiendo experiencias en equipos de trabajo, en el desarrollo de un proyecto utilizando para esto metáforas o analogías en forma secuencial y convirtiéndolos en modelos de ejecución, integrándolo en la cultura de la organización.

c.- La Combinación (explícito a explícito), es el proceso de crear conocimiento explicito proveniente de la compartición de este en toda la organización, donde otros empleados empiezan a asimilarlo, extenderlo, innovar y reformular su propio conocimiento tácito, como parte del conjunto de herramientas y recursos necesarios para hacer su trabajo.

d.- La Interiorización (explícito a tácito), es tomar el conocimiento explícito y convertirlo en tácito, es decir, crear procesos de entrenamiento, introducir prácticas de simulación con el propósito de crear nuevos paradigmas y modelos mentales compartidos acordes a los cambios del entorno. Esto inicia nuevamente la espiral del conocimiento, pero esta vez a un nivel superior donde las personas no reciben pasivamente el nuevo conocimiento, sino que lo interpretan activamente para que se 
ajuste a sus propias situaciones y perspectivas.

Figura 1. Modelo SECI. Socialización, Exteriorización, Combinación e Interiorización.

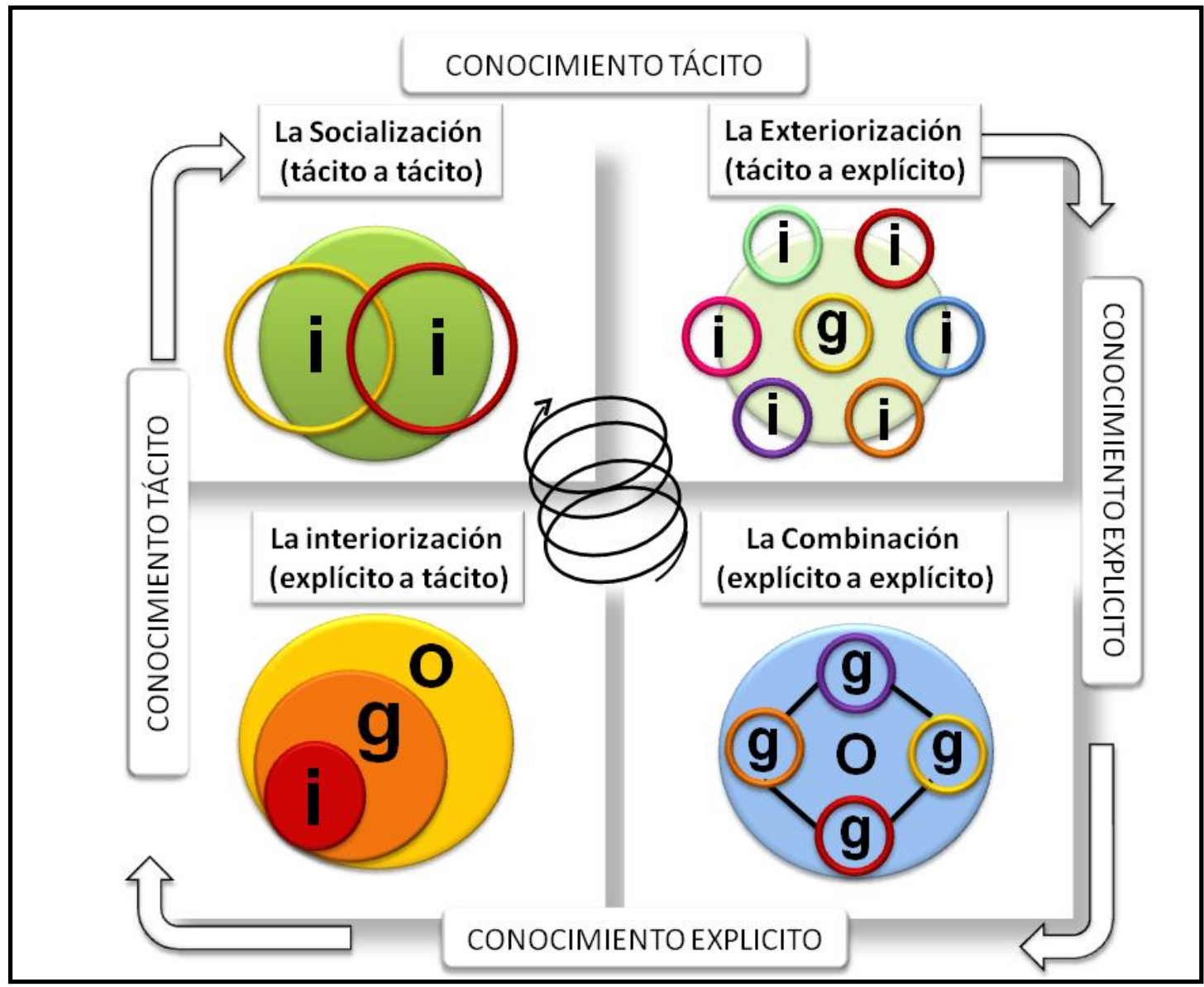

Fuente: Nonaka y Takeuchi (1995)

I= Individuo, G= Grupo, O=Organización

Nonaka y Takeuchi (1995), consideran que la estructura del conocimiento: "Está constituido en el conocimiento tácito, el cual tiene sus raíces en los más profundo de las acciones y la experiencia individual, así como los ideales, valores y emociones de cada persona." No es fácil planificar a través del lenguaje formal, el conocimiento tácito, por lo que resulta difícil transmitir y compartirlo con otro, la intuición, las ideas y las corazonadas subjetivas, son parte del individuo, este intelecto deja de ser individual y pasar a ser conocimiento explícito, siendo aquel que puede expresarse con palabras y números, sólo en ese instante, con una comunicación pertinente se puede transmitir y compartir los datos y procedimientos, para un mejor desempeño laboral, calidad de servicio, con un ambiente idóneo donde todos convergen sin distinciones ni rechazos.

La estructura del conocimiento, está constituido en el conocimiento tácito, el cual 
tiene sus raíces en los más profundo de las acciones y la experiencia individual, así como los ideales, valores y emociones de cada persona. No es fácil planearlo a través del lenguaje formal, por lo que resulta difícil transmitirlo y compartirlo con otro, la intuición, las ideas y las corazonadas subjetivas, son parte del individuo, este intelecto deja de ser individual y pasar a ser conocimiento explícito, siendo aquel que puede expresarse con palabras y números, puede transmitirse y compartirse fácilmente en forma de datos, fórmulas científicas, procedimientos o principios universales (Nonaka y Takeuchi, 1995).

En una economía donde lo seguro es la incertidumbre, la única fuente de ventaja competitiva duradera y segura es el conocimiento, cuando los mercados cambian, las tecnologías proliferan, los competidores se multiplican y los productos se vuelven obsoletos casi de un día para otro, las empresas exitosas son las que consistentemente crean nuevo conocimiento, lo diseminan ampliamente en toda la organización y lo incorporan rápidamente en nuevos productos y tecnologías, cuyo único propósito es la innovación continua.

El enfoque más holístico del conocimiento que se aplica en muchas empresas japonesas, se basa también en otra idea fundamental: una empresa no es una máquina, sino un organismo viviente, en forma muy semejante a una persona, puede tener un sentido colectivo de identidad; es el equivalente organizacional del autoconocimiento, una comprensión compartida de lo que la empresa representa, hacia donde va, el tipo de mundo en el que quiere vivir y lo más importante como convertirlo en realidad.

La conversión de los datos en información y luego en conocimientos es posiblemente la única fuente de competitividad sostenible, donde las organizaciones buscan el medio para hacer del conocimiento disponible un medio para incrementar su eficiencia, para estimular la innovación, para fundamentar la toma de decisiones y para elevar la eficacia, puesto que la transformación del conocimiento es un proceso social que depende de la cultura social de la organización, es decir, la capacidad del individuo de compartir la información, la disposición de experimentar y trabajar con otros para sus propios procesos de generar valor agregado.

La espiral del conocimiento no es un proceso lineal y secuencial, sino exponencial y dinámico, que parte del elemento humano y de su necesidad de contrastar, validar sus ideas y premisas; de esta forma el individuo a través de la experiencia crea conocimiento tácito, el cual conceptualiza, convirtiéndolo en explícito individual, solo cuando se logra compartir a través del diálogo fluido con otro o varias personas que forman parte de un departamento u organización, se convertirá en conocimiento 
explícito social, una vez alcanzada esta etapa de comunicación se debe interiorizar las experiencias, ideas o acciones de otras personas, lo cual genera una transmisión y percepción cíclica, en donde la comunicación es un factor primordial.

Su objetivo es incrementar los niveles de eficiencia y efectividad dentro de una organización, este proceso se traslada a través de la integración adecuada de los recursos humanos, las actividades y procedimientos que fundamentan la gestión del conocimiento. La retroalimentación que proporcionan la asignación adecuada de tareas y la evaluación objetiva del personal puede contribuir en gran medida a la satisfacción laboral, al aumento del desempeño de los empleados y al incremento de su sentido de pertenencia en las unidades de información, así como al mejoramiento de las relaciones entre subordinados y autoridades, en circunstancias que se caracterizan por la necesidad de cambio y la adaptación a nuevos contenidos y productos.

\section{Discusión}

Los resultados que se presentan a continuación son el reflejo de las indagaciones e investigaciones realizadas a través de la aplicación del instrumento de recolección de información hecho al personal que labora en las Bibliotecas de la Universidad Nacional Abierta Centro Local Falcón Sede Coro, las Unidades de Apoyo Capatárida, Churuguara y Punto Fijo; quienes son los responsables y administradores del recinto bibliotecario, en donde se especifican por dimensiones referidas al Modelo de Socialización, Exteriorización, Combinación e Interiorización ( $\mathrm{SECl}$ ), por lo tanto, los cambios producidos en la sociedad de la información implantan nuevas exigencias, llevando a reflexionar en las necesidades de formación de los recursos humanos de las Bibliotecas del Centro Local Falcón, para su desarrollo y aplicación, se estructuro en cuatro fases:

Fase I. Socialización del personal de las bibliotecas del Centro Local Falcón, en la cual se establece una comunicación horizontal entre los sujetos en estudio para obtener de ellos su conocimiento tácito e ir desarrollando un ambiente de intercambio de experiencias. 
Gráfico n: Socialización

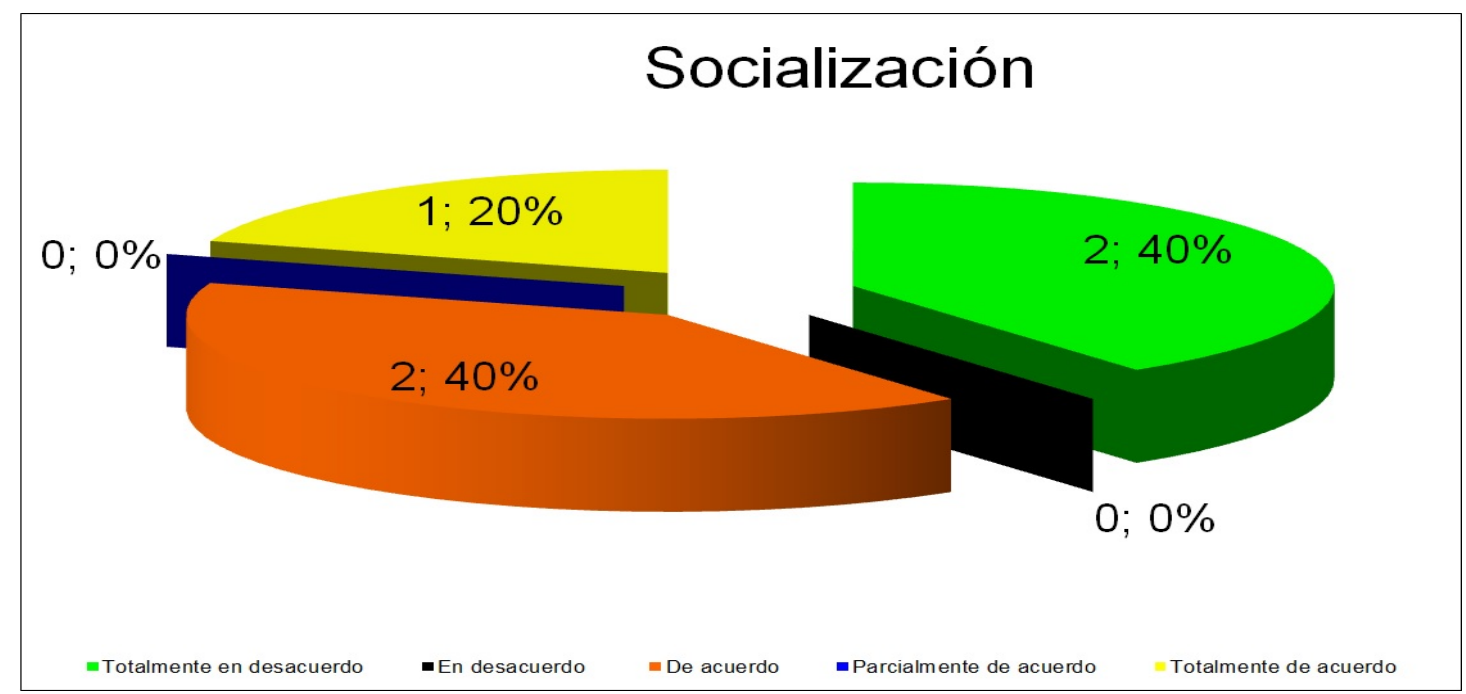

Fuente: Elaboración propia.

Moros (2003), argumenta: "la información como proceso de transformación de los datos, tiene importancia para cualquier institución y para preservar en el futuro la prestación de un mejor servicio de información, que a su vez permita mejorar y/o definir el conocimiento".

El resultado obtenido, determinó que 40\% está de acuerdo, 40\% totalmente en desacuerdo y $20 \%$ de acuerdo. Por lo tanto, las limitaciones presupuestarias para el traslado y supervisión de las Unidades de Apoyo, ha truncado la comunicación fluida y directa entre el supervisor y los responsables de las bibliotecas, a su vez se recurre a los medios tecnológicos para mantener una comunicación entre las partes, en la medida de lo posible, con la limitante del acceso a las computadoras y la conexión de banda ancha de internet para optimizar la comunicación en tiempo real, adicionalmente, el personal de las bibliotecas considera que su conocimiento y aprendizaje ha sido producto del ensayo y error, por años de experiencia dentro del recinto bibliotecario y determinando las necesidades de lectura de los usuarios en general lo que permitirá fortalecer la estructura organizativa, de los sistemas de información bibliotecarios, la comunicación y la interacción del personal como fuente primaria para el desarrollo organizacional dentro de la Universidad Nacional Abierta.

Fase II. Externalización del personal de las bibliotecas del Centro Local Falcón, una vez concretada la apreciación de todos los miembros de las bibliotecas, se procedió a esbozar el conocimiento explícito obtenido de aquellos con más pericia y años en la institución combinándolo con otros que tienen más conocimiento documental. 
Gráfico n²: Externalización

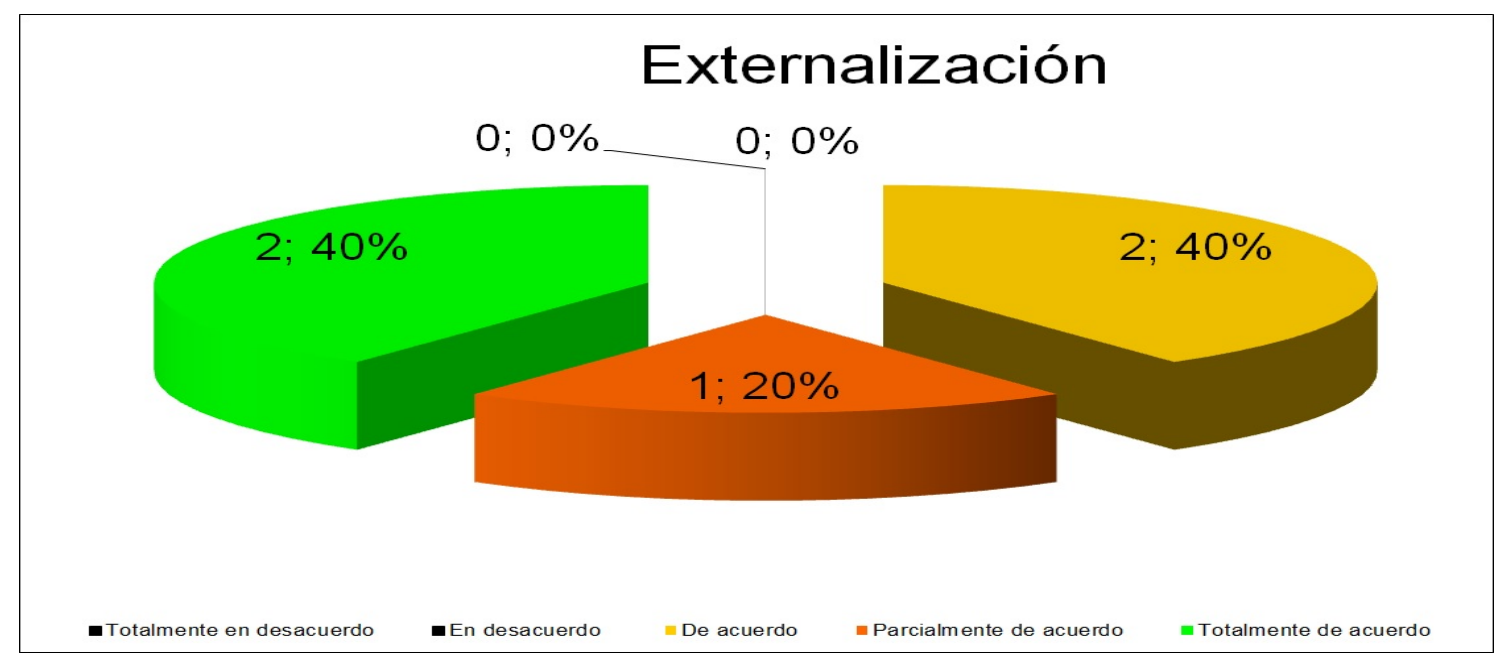

Fuente: Elaboración propia.

Matus Sepúlveda (2002), determino que: "el principal problema en las bibliotecas universitarias, está en torno a los recursos humanos, son insuficientes en la mayoría de las bibliotecas, ya que generalmente se han incrementado los recursos de información e incluso los materiales, pero se han mantenido o disminuido el personal, esto llevará irremediablemente a un menoscabo de la calidad del servicio que se ofrece y lo que consideramos más grave, terminará ocasionando un deterioro progresivo en la salud del personal motivado por una sobrecarga de trabajo".

Es evidente que la Universidad Nacional Abierta a mantenido un distanciamiento en cuanto al contrato de nuevo personal para laboral en las bibliotecas, el personal existente esta por años de servicio y ascenso dentro del recinto bibliotecario. Por lo tanto, esta situación ha repercutido en el personal como un factor indispensable para el logro de los procesos administrativos, necesarios para cumplir con los requerimientos exigidos para tal fin, la necesidad de trascender en el tiempo, que se apliquen y contribuyan a generar valor agregado para la UNA y la sociedad.

En las bibliotecas de la Universidad Nacional Abierta Centro Local Falcón, se promueven las estructuras técnicas para fomentar la externalización de habilidades y conocimientos entre sus miembros, todo esto conlleva a mantener un clima direccional bajo las pautas establecidas en las normas del Sistema Bibliotecario UNA (SIBIUNA), la aplicabilidad del Modelo SECI en los procedimientos de la UNA para con las bibliotecas, permitirá el mejoramiento continuo del personal.

Fase III. Combinación del personal de las bibliotecas del Centro Local Falcón, el 
resultado obtenido entre el personal que labora en las bibliotecas ha permitido fortalecer, acrecentar las técnicas y lineamientos con el objetivo de ofrecer una calidad de servicio dentro del recinto bibliotecario.

Gráfico nº: Combinación

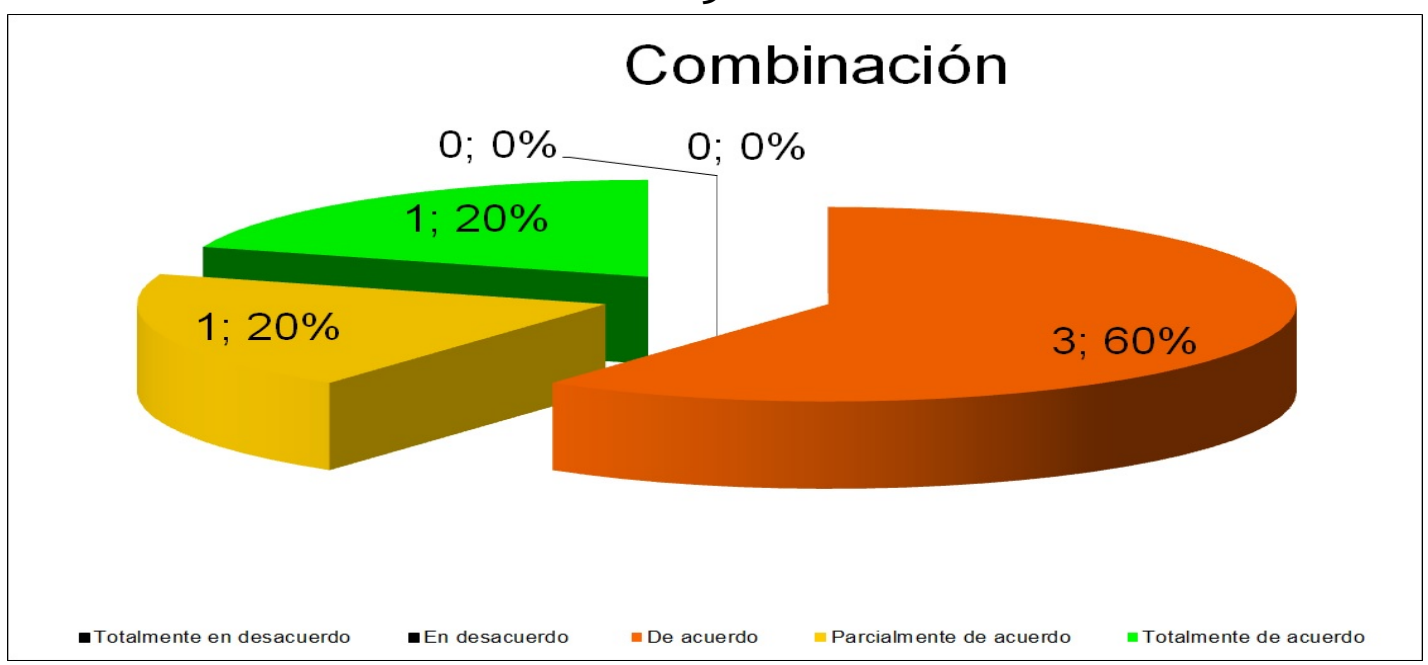

Fuente: Elaboración propia.

Choo (1998), argumenta:

"la búsqueda de información es el proceso humano y social a través del cual el conocimiento se vuelve útil para un individuo o grupo, siendo una variación en el estado de conocimiento para la selección y el procesamiento de la información con el fin de responder a una pregunta, solucionar un problema, tomar una decisión o comprender una situación".

Inicialmente la descripción de cargos del recurso humano de las bibliotecas del Centro Local Falcón, es un factor determinante en la aplicación de sus funciones dentro de la institución ya que al tener asignaciones específicas permite que sus tareas se cumplan con eficacia. Sin embargo, la ausencia de recursos, se agotan y llegan al condicionamiento de realizar otras tareas por la ausencia de personal, lo cual conlleva a la pérdida de rendimiento laboral y desmotivación.

Es importante resaltar que las Unidades de Recursos Múltiples, por pertenecer a la Universidad Nacional Abierta, donde su modalidad educativa formativa es a distancia, la combinación del personal y los servicios presentan una dimensión especial, utilizando métodos de comunicación ya sea por medios electrónicos, estos deben ir en función de las necesidades de sus usuarios, así como también el acceso al material bibliográfico, procurando que sea suficiente para atender a la totalidad de los usuarios, gestionando que los productos y servicios que se prestan sean pertinentes, eficientes y eficaces. 
Fase IV. Interiorización del personal de las bibliotecas del Centro Local Falcón, se refuerza el aprendizaje cognoscitivo de cada uno de los miembros de las bibliotecas con la finalidad de que el conocimiento adquirido crezca exponencialmente y sea aplicado en su entorno laboral. Este proceso del modelo SECl es cíclico, está constantemente transformando los datos en información, luego en conocimiento y en inteligencia aplicada a las bibliotecas del Centro Local Falcón de la Universidad Nacional Abierta.

Gráfico nº $^{\circ}$ : Interiorización

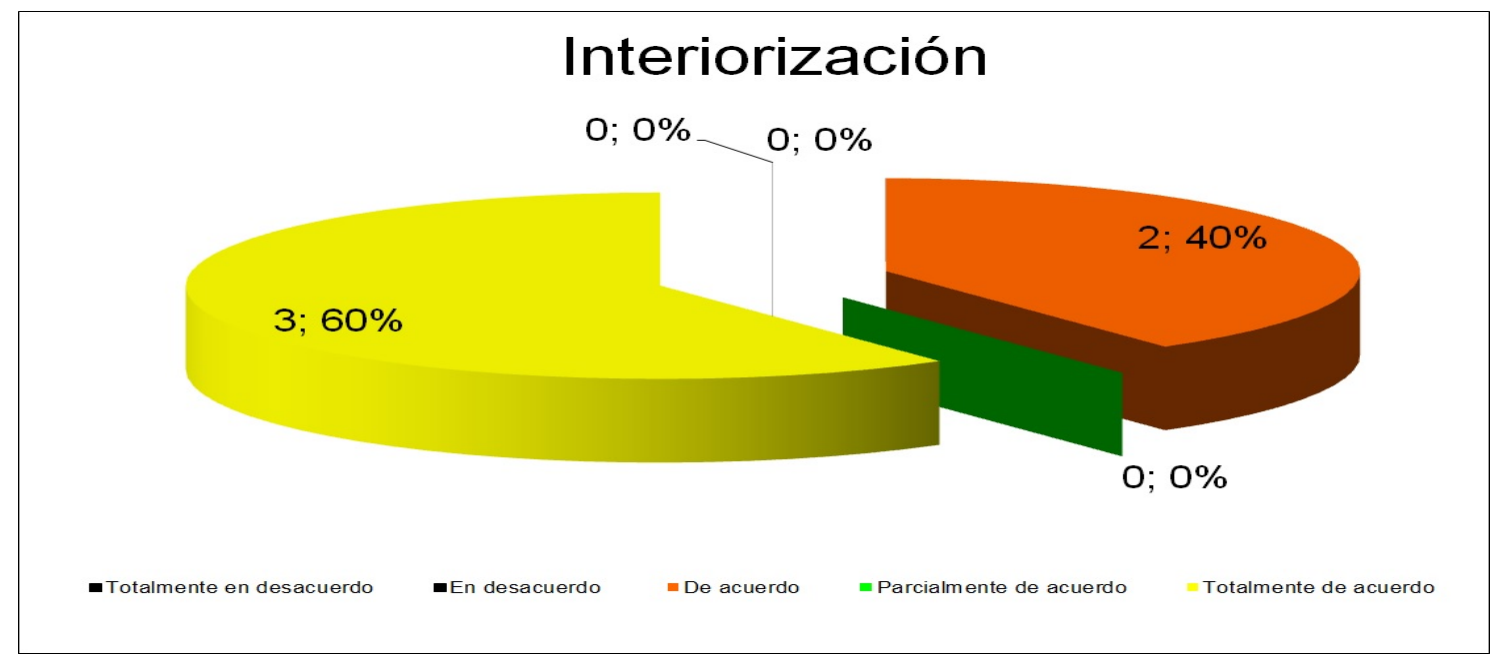

Fuente: Elaboración propia.

Páez Urdaneta (1992), argumenta:

Las decisiones son planificadas en el Centro de Recursos Múltiples (CRM) de la Universidad Nacional Abierta y luego emanadas a las coordinaciones de las bibliotecas a nivel nacional de la Universidad Nacional Abierta, los requerimientos, peticiones y demás vicisitudes que se presentan en las bibliotecas del Centro Local Falcón, son enviadas al CRM a la espera de una respuesta clara y concisa. Esta información es considerada como un recurso importante que se gestiona como tal, siendo un instrumento de control en la toma de decisiones a cualquier nivel.

El Centro de Recursos Múltiples (CRM) de la Universidad Nacional Abierta, transmite de forma clara y exigen mejor productividad en las funciones del empleado, con más carga de trabajo y en plazos breves, estableciendo las normativas y reglamentos que se deben realizar en los recintos bibliotecarios. La creciente complejidad del proceso de toma de decisiones, la presión en la demanda de respuestas urgentes a problemas complejos, conlleva a que el personal de las bibliotecas del Centro Local Falcón deban ser personas capacitadas, intuitivas, rápidas, creativas, con mentalidad abierta para 
que les permita actuar de forma práctica y concreta a la hora de tomar decisiones. Este factor humano, es determinante en cuanto a los conocimientos, habilidades, experiencias, capacidades, que deben ser desarrolladas mediante su capacitación antes o durante su desempeño profesional y laboral.

\section{Conclusión}

Prieto Figueroa (2006), argumenta: "En un mundo que se transforma rápidamente, los hombres han de estar en una constante actitud de adaptación y aprendizaje para poder actuar dentro de nuevas situaciones creadas".

En este orden de ideas, los cambios generados en el entorno de las bibliotecas del Centro Local Falcón, están contemplados hacia una nueva postura con respecto a la cultura que poseen las personas, siendo esta la creciente adecuación del individuo a las condiciones de medio donde labora, lo que conforma una identidad armónica cuando la experiencia se vuelve idea, la necesidad se transforma en técnica y en arte, adecuando de forma permanente los conocimientos y las habilidades de cada talento humano, para permitirle adaptarse a nuevas situaciones, tanto en el plano laboral, como en el social, y esto sólo es posible por medio de la gestión del conocimiento.

Sin embargo, las restricciones presupuestarias, las presiones institucionales y la preocupación creciente por la calidad han obligado a las bibliotecas del Centro Local Falcón a plantearse, cada vez con mayor frecuencia, el reto de conocer y valorar su propio funcionamiento, con miras a mejorar sus niveles de beneficio y eficacia, que permitan valorar el crecimiento cíclico de estas unidades de información de un modo continuo y normalizado.

Consolidar la contratación personal capacitado, bibliotecólogos, especialistas en información, asistentes, técnicos en información y documentación capaces de asumir y comprometerse en el desarrollo de una estructura que dé respuesta a usuarios ávidos de información y capaces de participar en procesos de asimilación tecnológica. Por lo tanto, la disminución de cargas laborales, es una tarea que ha dejado de ser opcional para transformarse en obligatoria, delegar y distribuir funciones o, por lo menos hacer el intento de ser escuchados en contra de algunos retrógrados. Este camino es largo y ya es obsoleto pensar en individualismo. Es oportuno destacar que los servicios bibliotecarios se fundamentan en cuatro bases muy sólidas:

En primer lugar, la formación, desarrollo y mantenimiento de las colecciones, conformado por medios impresos y electrónicos, las mismas deben responder a los objetivos de la institución a la cual sirven y a las necesidades de información de los usuarios, de la calidad del fondo bibliográfico depende el éxito de la biblioteca; 
En segundo lugar, personal capacitado, bibliotecólogos, especialistas en información, administradores, informáticos, técnicos en información y documentación capaces de asumir y comprometerse en el desarrollo de una estructura que dé respuesta a usuarios ávidos de información, capaces de participar en procesos de asimilación tecnológica;

En tercer lugar, servicios ágiles, actualizados, en la que el usuario sea tratado como el centro y razón de ser de la biblioteca, donde sus demandas son satisfechas, tengan acceso a la información, se promueva la lectura y la investigación;

En cuarto lugar, tecnología, para permitir el acceso a la información con pertinencia y eficiencia en el momento que se le necesite. Aun cuando algunas bibliotecas universitarias están utilizando sistemas tecnológicos y algunas otras se encuentran incorporándolas, la situación es crítica por el retraso de varias generaciones en la historia tecnológica que crece aceleradamente, por la falta de equipos, software y fallas a nivel organizacional.

Moros (2003), afirma, "el objetivo primordial es enlazar, en la medida de lo posible, lo concerniente al conocimiento, como un todo, y relacionarlo, con diferentes procesos, desde la definición conceptual, hasta el conglomerado tecnológico conocido como sistemas basados en conocimiento". Su interés creciente para gestionarlo, ya que el conocimiento siempre ha sido necesario para el funcionamiento de las organizaciones y de las personas, para la toma de decisiones y para las relaciones intra e internacionales, los organismos gubernamentales y multilaterales.

El personal bibliotecario del Centro Local Falcón, participa voluntariamente en la formación de enseñanza-aprendizaje de los usuarios en general, demostrando una empatía con los demás miembros de las diferentes dependencias que asisten a las bibliotecas. Es pertinente señalar que el enfoque tradicional del personal como recurso de valor agregado ha generado una dirección sistémica, teniendo en cuenta el recurso humano, como protagonista de los procesos que involucran la gestión del conocimiento, el conjunto de patrimonios intelectuales, su cultura y valores, que permiten una visión aproximada, para describir los procesos y estructuras, con la finalidad de orientar las estrategias de la mano de las TIC, favoreciendo el acceso rápido a la información interna y externa, que necesiten para alcanzar sus objetivos estratégicos.

La característica primordial del rol del personal adscrito a las bibliotecas del Centro 
Local Falcón de la Universidad Nacional Abierta, ante la aplicación del Modelo SECI de Nonaka y Takeuchi, estaría canalizado en:

1.- El personal es responsable de apoyar las actividades de docencia, investigación y extensión realizada por las universidades.

2.- Deben gestionar la información (real y virtual) disponible, en beneficio académico y de los usuarios.

3.- El recurso humano, debe ser proactivo en las Bibliotecas en las que están adscritos, compartiendo y promulgando su visión, misión, objetivos, estrategias y políticas.

4.- Estar claros y consientes en apoyar los programas y planes de estudio, los proyectos de investigación, la docencia, en las necesidades de los usuarios a los cuales se les direcciona para acceder y utilizar los servicios de las bibliotecas diversificándose para adaptarse a dichas facetas universitarias, siempre en la búsqueda de mejorar el desempeño laboral del personal y obtener la optimización de sus tareas, coexistiendo una retroalimentación entre las partes involucradas en el desarrollo educativo.

5.- Fomentar la socialización, exteriorización, combinación e interiorización del personal de las Bibliotecas del Centro Local Falcón, promulgación del acceso a las colecciones e instalaciones, cuyo propósito es ayudar a sus usuarios en el proceso de transformar la información en conocimiento, así como ofrecer la asistencia para el uso y gestión de los servicios de información, aumentando la importancia del aspecto social del espacio de las bibliotecas.

\section{Referencias}

Berrocal Berrocal, F., \& Pereda Marín, S. (2001). Formación y gestión del conocimiento. Revista Complutense de Educación, Vol. 12(2), 639-656.

Choo, Ch. W. (1998). La organización inteligente: el empleo de la información para dar significado, crear conocimiento y tomar decisiones. Oxford University Press.

Matus Sepúlveda, G. (06 de 03 de 2002). TDX Tesis Doctorals en Xarxa. (U. d. Lleida, Productor) https://tdx.cat/handle/10803/8292\#page=1

Moros, Á. (2003). Evolución del concepto de conocimiento desde la pirámide informacional: propuesta de servicio de gestión del conocimiento científico para instituciones de educación superior. Universidad Carlos III. 
Nonaka, I., \& Takeuchi, H. (1995). The knowledge-creating company: how Japanese companies create the dynamics of innovation. http://www.sopper.dk/speciale/arkiv/ni95.pdf

Páez Urdaneta, I. (1992). Gestión de la inteligencia, aprendizaje tecnológico y modernización del trabajo informacional: retos y oportunidades. Instituto de Estudios del Conocimiento de la Universidad Simón Bolívar, Consejo Nacional de Investigaciones Científicas y Tecnológicas.

Prieto Figueroa, Luis Beltrán (2006). El estado docente. Fundación Biblioteca Ayacucho.

Rodríguez, Alberto (2012). Estrategias de gerencia comunitaria. Fundación de Escuela de Gerencia Comunitaria.

Universidad Pédagogica Experimental Libertador. (2016). Manual de Trabajos de Grado de Especialización y Maestría y Tesis Doctorales. En UPEL, Manual de Trabajos de Grado de Especialización y Maestría y Tesis Doctorales (5ta ed.). UPEL.

\section{Fernando Antonio Salas Granado}

Licdo. En Bibliotecología - Universidad Central de Venezuela - UCV

MSc. Gerencia de Recursos Humanos - Universidad Nacional Experimental Rafael María Baralt - UNERMB

Experto en E-Learning - Fundación para la Actualización Tecnológica de Latinoamérica - FATLA

Coordinador de Biblioteca - Universidad Nacional Abierta - Centro Local Falcón ¿Cómo afrontar tus trabajos con ayuda de la Biblioteca? - Universidad de Murcia ¿Cómo diseñar un Plan de Comunicación? - Universidad Autónoma de Occidente Liderazgo e Inteligencia Emocional - MCA Business \& Postgraduate School Técnicas para la escritura creativa - MCA Business \& Postgraduate School Narrativa Digital - Universidad Nebrija

La biblioteca vacía. El tránsito de la cultura escrita a la cultura digital - Universidad Carlos III de Madrid

SEO: posicionamiento natural en buscadores - Vic - Universidad Central de Cataluña. 